Editor's Note: These short reviews of a recent paper in the Journal, written exclusively by graduate students or postdoctoral fellows, are intended to mimic the journal clubs that exist in your own departments or institutions. For more information on the format and purpose of the Journal Club, please see http://www.jneurosci.org/misc/ifa_features.shtml.

\title{
Mind the GAP: A Role for Neurofibromin in Restricting Axonal Plasticity
}

\author{
Andrew D. Gaudet and Leanne M. Ramer \\ Department of Zoology and International Collaboration on Repair Discoveries, The University of British Columbia, Vancouver, British Columbia, \\ Canada V6T 1 Z4
}

Review of Romero et al. (http://www.jneurosci.org/cgi/content/full/27/8/2124)

The axons of dorsal root ganglion (DRG) neurons project to cutaneous and soft tissue targets in the periphery, and centrally to the spinal cord and brainstem. The peripheral process can regenerate and reconnect with targets after injury, but the central process cannot regenerate beyond the PNS-CNS interface [the dorsal root entry zone (DREZ)]. The differential cellular responses to peripheral or dorsal root injury have contributed to our understanding of extrinsic and intrinsic barriers to regeneration in the adult CNS. In an interesting study published recently in The Journal of Neuroscience, Romero et al. (2007) used the dorsal root injury (DRI) model to determine whether neurofibromin affects axonal growth or sprouting in the CNS after injury. Neurofibromin, encoded by the neurofibromatosis type 1 (Nf1) tumor suppressor gene, contains a GTPase-activating protein (GAP)-related domain, which negatively regulates Ras GTPase activity and thereby prevents downstream Ras signaling (Fig. 1a) (Schubbert et al., 2007). Given that Ras

Received March 21, 2007; revised April 20, 2007; accepted April 21, 2007. A.D.G. and L.M.R. were supported by the Natural Sciences and Engineering Council of Canada and the Michael Smith Foundation for Health Research. We thank Drs. Matt Ramer and Andrei Krassioukov for their supervision and support. We also appreciate the discussion stimulated by colleagues that attend weekly Ramer/Tetzlaff journal club meetings.

Correspondence should be addressed to Andrew D. Gaudet, Department of Zoology and International Collaboration on Repair Discoveries, The University of British Columbia, 6270 University Boulevard, Vancouver, British Columbia, Canada V6T 1Z4. E-mail: adgaudet@interchange.ubc.ca.

DOI:10.1523/JNEUROSCI.1277-07.2007

Copyright $\odot 2007$ Society for Neuroscience $\quad$ 0270-6474/07/275533-02\$15.00/0 activation within neurons is associated with neurite elongation and cell survival (Fig. 1b), Romero et al. (2007) hypothesized that neurofibromin might suppress regeneration and/or sprouting of primary afferents after dorsal root injury.

The authors used a $L a c Z$ reporter gene to characterize the Cre-mediated inactivation of $N f 1$ in DRGs of Nf1flox/flox/ Synapsin I-Cre (Nf1SynIKO) mice, which they had generated previously (Zhu et al., 2001). Cre was expressed in the majority of large-diameter tropomyosin-related kinase $\mathrm{C}$ ( TrkC)-expressing proprioceptive DRG neurons, but not in calcitonin gene-related peptide (CGRP)-immunoreactive small-diameter nociceptors [Romero et al. (2007), their Fig. 1 (http:// www.jneurosci.org/cgi/content/full/27/8/ $2124 / F 1)]$. There was also a subset of LacZ-positive cells that did not express either CGRP or TrkC.

To determine whether conditional knock-out of $N f 1$ affected recovery of proprioception after DRI, the authors tested for the recovery of abductor muscle function, and performed grid walking tests and paw print analyses [Romero et al. (2007), their Figs. 3 (http://www.jneurosci.org/cgi/content/full/27/8/2124/F3), 4 (http://www.jneurosci.org/cgi/content/ full/27/8/2124/F4)]. Control DRI animals exhibited proprioceptive deficits that did not recover during the test period (up to $32 \mathrm{~d}$ postinjury). In contrast, $N$ f1SynIKO mice displayed accurate paw placement and regained normal toe spread by $24 \mathrm{~d}$ postinjury. Importantly, the extent of recovery was equivalent between Nf1SynIKO mice that received lumbar 4 (L4)/L5 crush DRI (regeneration possible), and Nf1SynIKO mice in which the same roots were cut and ligated (precluding regeneration). Thus, a mechanism other than regeneration was responsible for recovery. The authors provided histological confirmation that proprioceptive afferents did not regenerate in Nf1SynIKO mice [Romero et al. (2007), their supplemental data 6 (http://www. jneurosci.org/cgi/content/full/27/8/2124/ DC1)].

One possible explanation for recovery in Nf1SynIKO mice is that axons from uninjured, adjacent DRGs show enhanced sprouting in the absence of neurofibromin signaling. Explant cultures of Nf1SynIKO DRG neurons exhibited increased axonal outgrowth in vitro [Romero et al. (2007), their Fig. 2 (http:// www.jneurosci.org/cgi/content/full/27/8/ 2124/F2)]. To establish whether sensory neurons lacking neurofibromin had enhanced growth capacity in vivo, Romero et al. (2007) examined immunohistochemical localization of CGRP and cholera toxin B (CTB; a tracer used to visualize large-diameter afferents/motoneurons) in the gray matter of the lumbosacral spinal cord. The distribution of CGRP and CTB labeling was similar in the two strains without injury [Romero et al. (2007), their Fig. 5 (http://www.jneurosci.org/ cgi/content/full/27/8/2124/F5)]. DRI- 
induced sprouting from adjacent segments did occur in Nf1SynIKO mice, whereas CGRP-expression and CTBtransporting axons were nearly eliminated in the dorsal horn in control mice (unidentified time point). There was also an increased density of CTB tracing in the deep layers of the L4-L5 dorsal horn of Nf1SynIKO mice [Romero et al. (2007), their Fig. 6 (http://www.jneurosci.org/ cgi/content/full/27/8/2124/F6)]. In the representative images, it seemed to us that CGRP-positive fibers may have sprouted as well, although the authors say not. Thus, neurofibromin suppresses the ability of intact DRG axons to sprout within the spinal cord in response to deafferentation, and this sprouting may contribute to functional recovery after DRI.

Cre recombinase is expressed in many CNS neurons in Nf1SynIKO mice. To determine whether conditional knock-out of $N f 1$ affected sprouting by mechanisms intrinsic or extrinsic to DRGs, Romero et al. (2007) cocultured control and Nf1SynIKO spinal cords and DRGs in Matrigel [Romero et al. (2007), their Fig. 7 (http://www.jneurosci.org/cgi/content/ full/27/8/2124/F7)]. Neurite outgrowth from Nf1SynIKO DRG neurons was greater than control DRGs and, interestingly, outgrowth from Nf1SynIKO DRG neurons was further increased in the presence of Nf1SynIKO spinal cords. This suggests that conditional knock-out of $N f 1$ in DRG and spinal cord neurons synergistically enhances axonal sprouting from DRGs.

In their final experiment, the authors set out to establish which signaling pathways were affected by conditional Nf1 deletion. Using Western blots, they found a significantly higher ratio of activated (phosphorylated) Erk to total Erk protein in the CNS and DRG of Nf1SynIKO mice. Although the authors suggest that the phosphatidyl inositol 3 kinase-Aktglycogen synthase kinase $3 \beta$ (GSK3 $\beta$ ) cascades may also be involved in the enhanced sprouting, there was no significant difference in the activation of either Akt or GSK $3 \beta$ in the DRGs of Nf1SynIKO mice. Thus, endogenous neurofibromin likely represses axon sprouting by inhibiting Ras activation of the mitogen-activated protein (MAP) kinase kinase (MEK)-Erk MAP kinase pathway.

In summary, Romero et al. (2007) define a role for neurofibromin in the suppression of axonal plasticity after DRI. Although conditional knock-out of $N f-1$

a
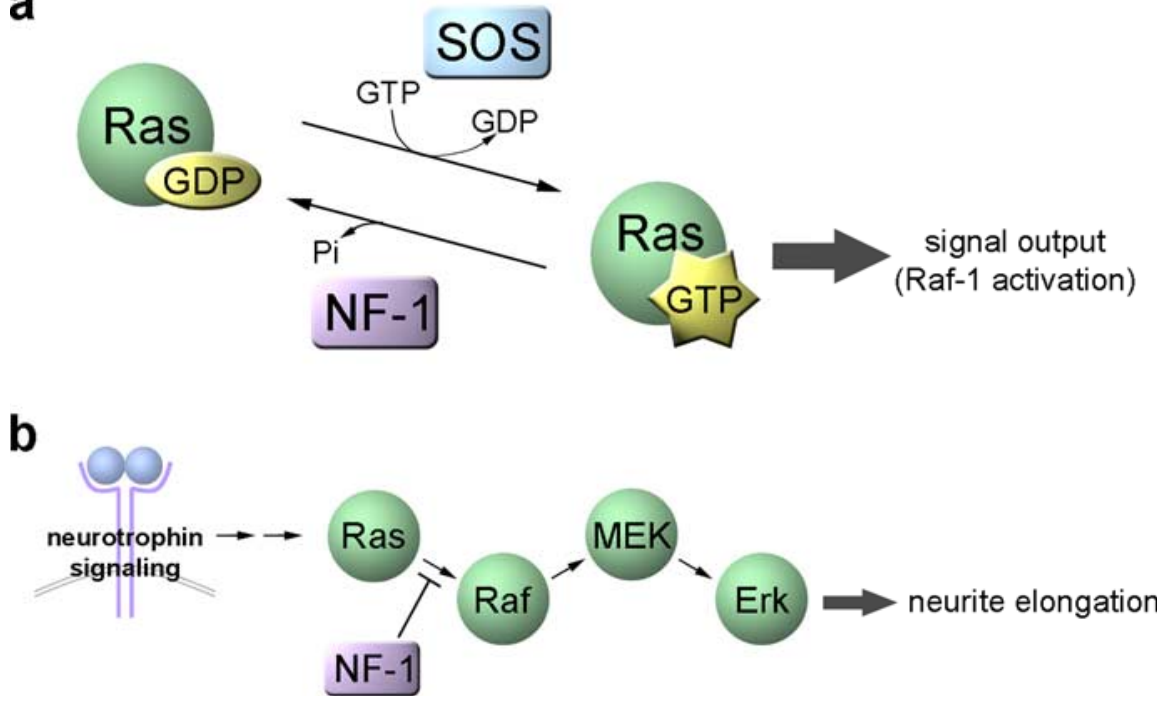

Figure 1. Role of neurofibromin (NF-1) in the regulation of intracellular Ras signaling within DRG neurons. $\boldsymbol{a}$, When activated, the guanosine-nucleotide exchange factor Son-of-Sevenless (SOS) displaces GDP from inactive Ras. This allows GTP, which is abundant in the cytosol, to bind Ras passively. GTP-bound Ras can then activate downstream effectors such as Raf-1. The GAP neurofibromin promotes Ras to convert GTP to GDP and thereby prevents downstream signaling (Schubbert et al., 2007). $\boldsymbol{b}$, Although Ras activates various pathways, its involvement in the MAP kinase pathway has been studied most intensively. This pathway involves the dimerization of receptor tyrosine kinases (e.g., Trk receptors) that then activate SOS. After Ras activation, a phosphorylation cascade ensues, with subsequent activation of Raf-1, MEK, and Erk-1 and Erk-2, which ultimately results in the transcription of genes encoding Ets family transcription factors (Mor and Philips, 2006). Romero et al. (2007) showed that neurofibromin negatively regulates activation of the MEK-Erk pathway, which may underlie its plasticity-limiting influence on spared sensory projections to the deafferented spinal cord.

was not sufficient for regeneration of injured DRG axons across the gliotic scar at the DREZ, it did allow uninjured axons from adjacent DRGs to sprout ectopically into deafferented segments. This DRIinduced central sprouting is likely responsible for the recovery of proprioceptive function observed in Nf1SynIKO mice.

It is well accepted that spontaneous recovery after neural trauma depends on plasticity of spared neurons. As a result of the inherent challenges in promoting functionally relevant regeneration in the adult CNS, plasticity of spared systems may represent a realistic substrate for stimulating functional recovery. Because neurofibromin specifically limited injuryinduced plasticity of spared neurons, it may be a therapeutic target, with the caveat that tissue-specific deletions of $\mathrm{Nf1}$ will be required to delineate the intrinsic and extrinsic effects.

It will be interesting to know whether sprouting of peripheral branches of DRG neurons has a role in the recovery of proprioception after DRI in NfISynIKO mice. The GAP-related domain of neurofibromin is not sufficient to rescue neural crest deficits in Nf1 mutant mice (Ismat et al., 2006). Thus, it seems that other domains are required for the regulation of neurofibromin or for targeting to acti- vated Ras. It will also be interesting to study DRI-induced axonal plasticity in mice that express Cre with more spatiotemporal resolution [e.g., SPRR1A (small proline-rich repeat protein $1 \mathrm{~A}$ ), which is expressed in DRG neurons after axonal injury (Bonilla et al., 2002)].

\section{References}

Bonilla IE, Tanabe K, Strittmatter SM (2002) Small proline-rich repeat protein $1 \mathrm{~A}$ is expressed by axotomized neurons and promotes axonal outgrowth. J Neurosci 22:1303-1315.

Ismat FA, Xu J, Lu MM, Epstein JA (2006) The neurofibromin GAP-related domain rescues endothelial but not neural crest development in Nf1 mice. J Clin Invest 116:2378-2384.

Mor A, Philips MR (2006) Compartmentalized Ras/MAPK signaling. Annu Rev Immunol 24:771-800.

Romero MI, Lin L, Lush ME, Lei L, Parada LF, Zhu Y (2007) Deletion of Nf1 in neurons induces increased axon collateral branching after dorsal root injury. J Neurosci 27:2124-2134.

Schubbert S, Bollag G, Shannon K (2007) Deregulated Ras signaling in developmental disorders: new tricks for an old dog. Curr Opin Genet Dev 17:15-22.

Zhu Y, Romero MI, Ghosh P, Ye Z, Charnay P, Rushing EJ, Marth JD, Parada LF (2001) Ablation of NF1 function in neurons induces abnormal development of cerebral cortex and reactive gliosis in the brain. Genes Dev 15:859-876. 